\title{
Motion Curve Optimization of Intermittent Conveyance Bottling Machine for Reducing Liquid Vibration
}

\author{
Ken'ichi Kanazawa ${ }^{1}$, Keiji Yasuya ${ }^{1}$, Norihiko Kato ${ }^{1}$, Ken' $^{\prime}$ ichi Yano $^{1}$ and Tatsuhiro Nakada ${ }^{2}$
}

\begin{abstract}
Recently, the need to accelerate the operating cycle of bottling machines while maintaining low operation cost has arisen. However, conveying bottles at high speed without spilling the liquids inside requires advanced techniques for vibration suppression control. In this paper, we optimize the motion curve for an intermittent conveyance bottling machine by using computational fluid dynamics (CFD) simulation to decrease residual vibration at the surface of the liquid. Additionally, we propose a method for assigning magnitude relations to variables and defining the curve by using variable transformations.
\end{abstract}

\section{INTRODUCTION}

Recently, high-speed operation cycles for bottling machines and reduction of production costs have come to be required. A conventional approach to improving operation cycles involves increasing the number of bottles filled simultaneously. However, such an approach requires increasing the size of the bottling machine and leads to an increase in production cost. An alternative approach to improving operation cycles without increasing the cost is raising the speed of bottle conveyance. Toward that end, advanced techniques for suppressing vibration at the surface of liquids are required for conveying bottles at high speed without spilling the liquids.

In a previous study on vibration suppression control for surfaces of liquids, Yoshida et al. have proposed a transfer control method for a cylindrical container for liquids by regarding it as a spherical pendulum model [1]. However, since the shape of most actual bottles is not as simple as a cylindrical container for liquids, applying that method to an actual bottling machine is difficult. The development of a fluid flow model for a bottle with a complex shape can be aided by computational fluid dynamics (CFD). In this regard, we have previously used a CFD simulator to optimize the trajectory planning of a spoon that contains a liquid [2] and to optimize the flow of molten metal in casting [3], [4].

In the present study, we aim to obtain an optimized motion curve for an intermittent conveyance bottling machine by using CFD simulation to decrease residual vibration at the surface of the liquid. The motion curve is defined as a spline curve that interpolates several points and is optimized by using a real-coded genetic algorithm (RealGA) [5]. We also propose a method for assigning magnitude relations to variables for the coordinates of points by using variable transformations. The effectiveness of the optimized motion

${ }^{1}$ K. Kanazawa, K. Yasuya, N. Kato and K. Yano are with Department of Mechanical Engineering, Mie University, Kurimamachiya-cho, Tsu-city, 514-8507, Japan yanolab@robot.mach.mie-u.ac.jp

${ }^{2}$ T. Nakada is with Shibuya Kogyo Co., Ltd., Mamedahon-machi, Kanazawa-city, 920-8681, Japan

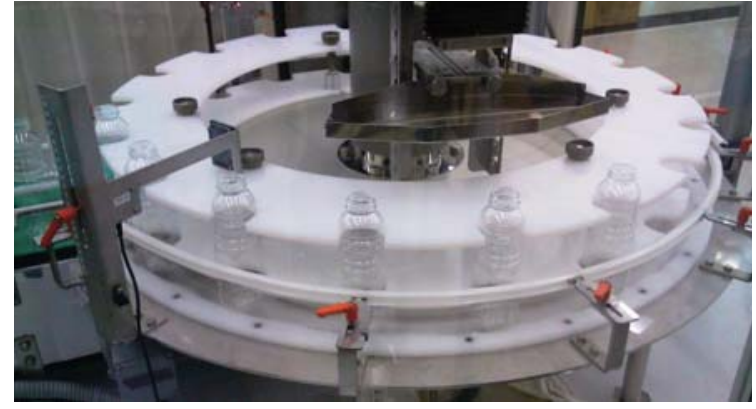

Fig. 1. Intermittent conveyance bottling machine

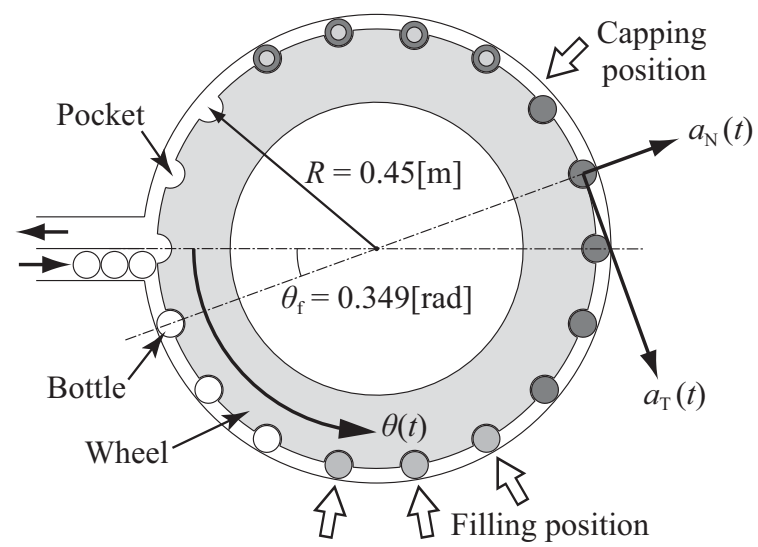

Fig. 2. Outline of the intermittent conveyance bottling machine

curve is demonstrated through experiments using an actual bottling machine.

\section{INTERMITTENT CONVEYANCE BOTTLING MACHINE}

An intermittent conveyance bottling machine and its outline are shown in Figs. 1 and 2 respectively, where "intermittent conveyance" indicates that the machine alternates conveyance and idling stages. The machine conveys bottles by rotating a wheel during the conveyance stage and caps the bottles during the idling stage. In this case, the machine fills three bottles with liquid simultaneously at every three cycles.

The rolling radius of the bottles is $R=0.45[\mathrm{~m}]$. Since the wheel has 18 pockets, the rotation angle per cycle is $\theta_{\mathrm{f}}=$ $20[\mathrm{deg}] \approx 0.349[\mathrm{rad}]$. A reference rotation angle $\theta_{\text {ref }}(t)$ of the wheel with respect to time is used as a reference input to the machine, where the relationship between $\theta_{\text {ref }}(t)$ and the actual rotation angle $\theta(t)$ of the wheel is expressed as 
follows:

$$
\mathcal{L}[\theta(t)]=\frac{1}{0.02 s+1} \mathcal{L}\left[\theta_{\text {ref }}(t)\right] .
$$

Since the wheel can rotate only to the left at any time due to a constraint of the machine, both $\theta_{\text {ref }}(t)$ and $\theta(t)$ must be weakly increasing functions.

When a bottle is conveyed according to $\theta(t)$, the relative accelerations acting on the liquid at the center of the bottle are the acceleration in the tangential direction $a_{\mathrm{T}}(t)\left[\mathrm{m} / \mathrm{s}^{2}\right]$, the acceleration in the normal direction $a_{\mathrm{N}}(t)\left[\mathrm{m} / \mathrm{s}^{2}\right]$, and the gravitational acceleration $a_{\mathrm{G}}=9.81\left[\mathrm{~m} / \mathrm{s}^{2}\right] . a_{\mathrm{T}}(t)$ and $a_{\mathrm{N}}(t)$ are expressed as follows:

$$
a_{\mathrm{T}}(t)=R \frac{d^{2} \theta(t)}{d t^{2}}=R \alpha(t)
$$

and

$$
a_{\mathrm{N}}(t)=R\left(\frac{d \theta(t)}{d t}\right)^{2}=R \omega(t)^{2},
$$

where $\omega(t)$ and $\alpha(t)$ are the angular velocity and the angular acceleration of the wheel, respectively. Since the magnitudes of steady $a_{\mathrm{N}}(t)$ and $a_{\mathrm{T}}(t)$ have a direct impact on the slope of the surface of the liquid, the respective maxima of $\omega(t)$ and $|\alpha(t)|$ that determine them must be considered in order to obtain $\theta(t)$. Additionally, since sudden changes in $a_{\mathrm{N}}(t)$ and $a_{\mathrm{T}}(t)$ strongly affect the behavior of the surface of the liquid, the angular jerk $j(t)$ must also be considered.

\section{DEFINITION OF MOTION CURVE}

\section{A. Modified Sine Curve}

A modified sine curve is one of the most commonly used motion curves. In this paper, we regard it as a criteria curve and use it for comparison with the optimized motion curve. A dimensionless modified sine curve is expressed as follows:

$$
\hat{\theta}_{\mathrm{M}}(t)=\left\{\begin{array}{r}
-\frac{1}{4(4+\pi)} \sin (4 \pi t)+\pi t, \\
\text { if } 0 \leq t<\frac{1}{8} \\
-\frac{9}{4(4+\pi)} \sin \left(\frac{4}{3} \pi t+\frac{\pi}{3}\right)+\pi t+2, \\
\text { if } \frac{1}{8} \leq t<\frac{7}{8} \\
-\frac{1}{4(4+\pi)} \sin (4 \pi t)+\pi t+4, \\
\text { if } \frac{7}{8} \leq t \leq 1 .
\end{array}\right.
$$

Both the first and second derivatives of $\hat{\theta}_{\mathrm{M}}(t)$ are continuous. A modified sine curve is characterized by relatively small maximum values of these derivatives.

\section{B. Definition of Motion Curve Based on the Spline Curve}

A reference motion curve $\theta_{\text {ref }}(t)$ is defined as a curve that features considerable flexibility with only a small number of variables from the perspective of optimization. Therefore, we define $\theta_{\text {ref }}(t)$ on the basis of a spline curve. However, since $\theta_{\text {ref }}(t)$ must be a weakly increasing function, we define the angular velocity curve $\omega_{\text {ref }}(t)$, which is obtained as the differential of $\theta_{\text {ref }}(t)$ with respect to time, by using a spline curve first and subsequently integrating $\omega_{\text {ref }}(t)$ and normalizing the result.
A dimensionless velocity curve $\hat{\omega}_{\text {ref }}(t)$ is shown in Fig. 3 . $\hat{\omega}_{\text {ref }}(t)$ is defined from (8) and (9) as a natural cubic spline curve [6] that interpolates the two endpoints $(0,0)$ and $(1,0)$, as well as $n$-pass points:

$$
P_{i}=\left(\tau_{i}, w_{i}\right),
$$

where

$$
0 \leq \tau_{1} \leq \tau_{2} \leq \cdots \leq \tau_{n} \leq 1
$$

and

$$
0 \leq w_{i} \leq 1
$$

for $i=1,2, \ldots, n$.

Further,

$$
\hat{\omega}_{\mathrm{ref}}(t)=\left\{\begin{array}{cc}
\omega_{0}(t), & \text { if } 0 \leq t \leq \tau_{1} \\
\omega_{1}(t), & \text { if } \tau_{1} \leq t \leq \tau_{2} \\
\vdots & \\
\omega_{n}(t), & \text { if } \tau_{n} \leq t \leq 1
\end{array}\right.
$$

and

$\omega_{i}(t)=A_{i}\left(t-\tau_{i}\right)^{3}+B_{i}\left(t-\tau_{i}\right)^{2}+C_{i}\left(t-\tau_{i}\right)+D_{i}$,

where

$$
\begin{aligned}
A_{i} & =\frac{1}{6 h_{i}}\left(z_{i+1}-z_{i}\right), \\
B_{i} & =\frac{z_{i}}{2} \\
C_{i} & =-\frac{h_{i}}{6} z_{i+1}-\frac{h_{i}}{3} z_{i}+\frac{1}{h_{i}}\left(w_{i+1}-w_{i}\right), \\
D_{i} & =w_{i}
\end{aligned}
$$

and

$$
h_{i}=\tau_{i+1}-\tau_{i},
$$

for $i=1,2, \ldots, n$.

$z_{i}$ in the above equations is obtained by solving the following equation:

$$
\begin{aligned}
& {\left[\begin{array}{cccccc}
1 & & & & & \\
u_{1} & h_{1} & & & \mathbf{O} & \\
h_{1} & u_{2} & h_{2} & & & \\
& h_{2} & u_{3} & h_{3} & & \\
& & \ddots & \ddots & \ddots & \\
& 0 & & h_{n-2} & u_{n-1} & h_{n-1} \\
& \mathbf{O} & & & h_{n-1} & u_{n} \\
& & & & & 1
\end{array}\right]} \\
& \times\left[\begin{array}{c}
z_{0} \\
z_{1} \\
z_{2} \\
\vdots \\
z_{n} \\
z_{n+1}
\end{array}\right]=\left[\begin{array}{c}
0 \\
v_{1} \\
v_{2} \\
\vdots \\
v_{n} \\
0
\end{array}\right]
\end{aligned}
$$

where

$$
u_{i}=2\left(h_{i}+h_{i-1}\right)
$$




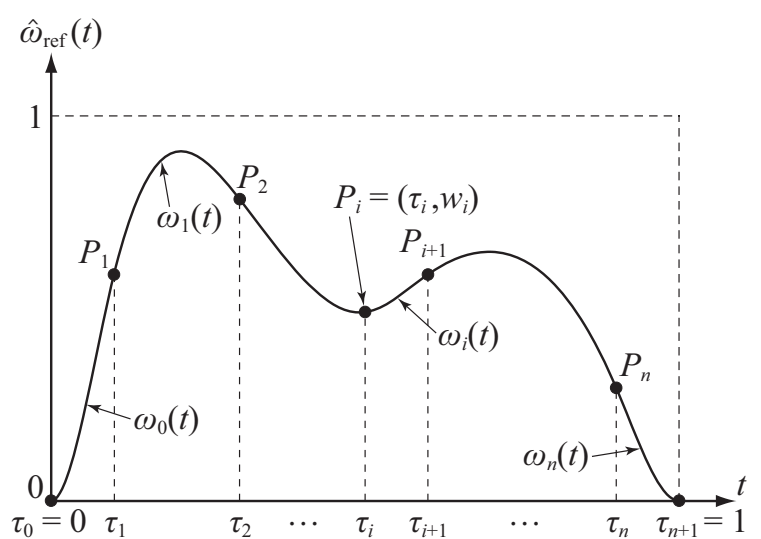

Fig. 3. Definition of a natural cubic spline curve

and

$$
v_{i}=\frac{6}{h_{i}}\left(w_{i+1}-w_{i}\right)-\frac{6}{h_{i-1}}\left(w_{i}-w_{i-1}\right) .
$$

We obtain a dimensionless reference motion curve $\hat{\theta}_{\text {ref }}(t)$ of the following equation by dividing the integral of $\hat{\omega}_{\text {ref }}(t)$ by its definite integral from 0 to 1 .

$$
\hat{\theta}_{\mathrm{ref}}(t)=\frac{\int \hat{\omega}_{\mathrm{ref}}(t) d t}{\int_{0}^{1} \hat{\omega}_{\mathrm{ref}}(t) d t} .
$$

Finally, when the rotation angle is $\theta_{\mathrm{f}}$ and the motion time is $t_{\mathrm{f}}$, the reference motion curve is generalized as follows:

$$
\theta_{\text {ref }}(t)=\theta_{\mathrm{f}} \cdot \hat{\theta}_{\text {ref }}\left(\frac{t}{t_{\mathrm{f}}}\right) \text {. }
$$

\section{Method for Transformation of Variables into Variables with Magnitude Relations}

The constraint conditions for the variables $\tau_{i}$ and $w_{i}$ which define $\theta_{\text {ref }}(t)$ are shown in (6) and (7), respectively. In particular, the upper and lower limits of $\tau_{i}$ are changed by the other variables. On the other hand, when a RealGA is used as an optimization algorithm, the upper and lower limits of $\tau_{i}$ must be defined as constants. One of the methods for applying a RealGA to an optimization problem with these constraint conditions is to sort internally each given $\tau_{i}$ by meeting the constraint conditions. However, there is a possibility that the performance of the RealGA degrades due to the relationship between variables (genotype), in which case their character (phenotype) becomes discontinuous. The other method involves defining $\tau_{i}$ as a constant rather than as a variable. In this case, a satisfactory optimization result might not be obtained because of the limited flexibility of $\theta_{\text {ref }}(t)$.

Thus, in the following we utilize a simple variable transformation to develop an equation of transformation which converts variable $x_{i}$ on $0 \leq x_{i} \leq 1$ into $\tau_{i}$ and which satisfies the constraint condition in (6). Assuming the precondition that if $x_{i}$ is a uniformly distributed random number, $\tau_{i}$ is also distributed uniformly in the region of (6).

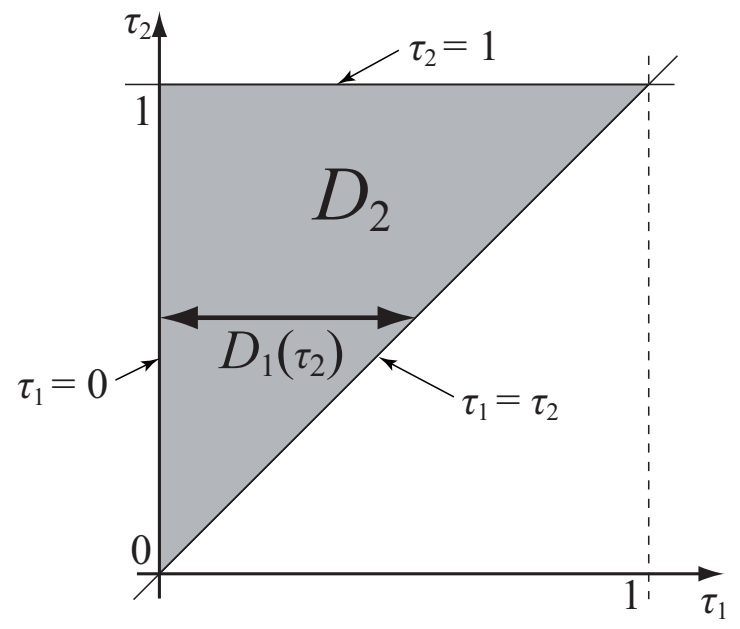

Fig. 4. Range of $D_{2}$ on a two-dimensional surface

Step 1. The case of $n=2 .:$ In (6), $0 \leq \tau_{1} \leq \tau_{2} \leq 1$. It is geometrically expressed in Fig. 4 as the triangular region $D_{2}$ surrounded by the lines $\tau_{1}=0, \tau_{1}=\tau_{2}$, and $\tau_{2}=1$, where the area of $D_{2}$ is $1 / 2$.

The condition that $\tau_{1}$ and $\tau_{2}$ are distributed uniformly in accordance with (6) is equivalent to the condition that the point $\left(\tau_{1}, \tau_{2}\right)$ is distributed uniformly in $D_{2}$. When $\tau_{2}$ takes an arbitrary value, assuming that the range of $\tau_{1}$ is $D_{1}\left(\tau_{2}\right)$, a uniform distribution of $\tau_{1}$ in $D_{1}\left(\tau_{2}\right)$ is realized by multiplying $x_{1}$ by $\tau_{2}$. As $\tau_{2}$ becomes smaller, $D_{1}\left(\tau_{2}\right)$ becomes smaller, and the distribution density of $\tau_{1}$ becomes higher in inverse proportion to $\tau_{2}$. Therefore, the distribution of the point $\left(\tau_{1}, \tau_{2}\right)$ can be made uniform in $D_{2}$ by setting the probability density function of $\tau_{2}$ to be proportional to $\tau_{2}$. We use inverse transform sampling to obtain an equation for transforming a variable into $\tau_{2}$ characterized by this probability density function.

Inverse transform sampling is a method for generating a random variable $\tau$ characterized by any probability density function $f(\tau)$. This method involves a random variable $x$ that is uniformly distributed on $[0,1]$, and uses the following equation:

$$
\tau=F^{-1}(x)
$$

where $F^{-1}(x)$ is the inverse function of $F(\tau)$ and $F(\tau)$ is the integral of $f(\tau)$ or the cumulative distribution function of $\tau$.

Since the probability density function of $\tau_{2}$ is proportional to $\tau_{2}$, and since the definite integral of $f_{2}\left(\tau_{2}\right)$ on $[0,1]$ must be 1 , then $f_{2}\left(\tau_{2}\right)$ is $f_{2}\left(\tau_{2}\right)=2 \tau_{2}$. Thus, $\tau_{1}$ and $\tau_{2}$ are obtained as follows by applying inverse transform sampling to $f_{2}\left(\tau_{2}\right)$ by using $x_{2}$ :

$$
\left\{\begin{array}{l}
\tau_{1}=x_{1} \tau_{2}=x_{1} x_{2}^{\frac{1}{2}} \\
\tau_{2}=x_{2}^{\frac{1}{2}} .
\end{array}\right.
$$

Step 2. The case of $n=3 .:$ In (6), $0 \leq \tau_{1} \leq \tau_{2} \leq \tau_{3} \leq$ 1. As in the case of step 1 , it is geometrically expressed, as shown in Fig. 5, as a tetrahedron-shaped region $D_{3}$ 


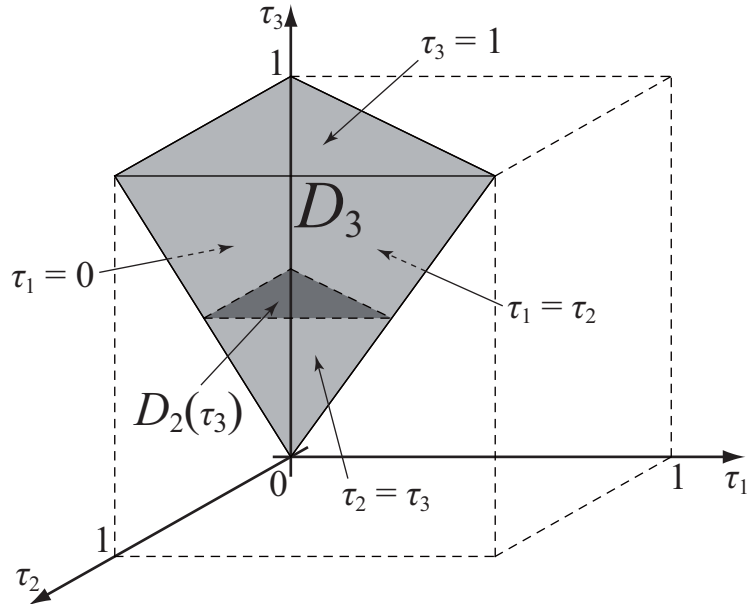

Fig. 5. Range of $D_{3}$ in three-dimensional space

surrounded by the planes $\tau_{1}=0, \tau_{1}=\tau_{2}, \tau_{2}=\tau_{3}$ and $\tau_{3}=1$, where the volume of $D_{3}$ is $1 / 6$.

When $\tau_{3}$ takes an arbitrary value, assuming that the range of $\left(\tau_{1}, \tau_{2}\right)$ is $D_{2}\left(\tau_{3}\right)$, a uniform distribution of $\left(\tau_{1}, \tau_{2}\right)$ in $D_{2}\left(\tau_{3}\right)$ is realized by multiplying the right-hand sides of (21) by $\tau_{3}$. Since $D_{2}\left(\tau_{3}\right)$ is a two-dimensional shape, the distribution density of $D_{2}\left(\tau_{3}\right)$ is inversely proportional to the square of $\tau_{3}$. Therefore, by assuming $f_{3}\left(\tau_{3}\right)=3 \tau_{3}^{2}$ (where $\int_{0}^{1} f_{3}\left(\tau_{3}\right)=1$ ) in order to set the probability density function of $\tau_{3}$ to be proportional to the square of $\tau_{3}$, the distribution of $\left(\tau_{1}, \tau_{2}, \tau_{3}\right)$ can be made uniform in $D_{3}$. Thus, $\tau_{1}, \tau_{2}$, and $\tau_{3}$ are obtained as follows by applying the inverse transform sampling to $f_{3}\left(\tau_{3}\right)$ by using $x_{3}$.

$$
\left\{\begin{array}{l}
\tau_{1}=x_{1} x_{2}^{\frac{1}{2}} \tau_{3}=x_{1} x_{2}^{\frac{1}{2}} x_{3}^{\frac{1}{3}} \\
\tau_{2}=x_{2}^{\frac{1}{2}} \tau_{3}=x_{2}^{\frac{1}{2}} x_{3}^{\frac{1}{3}} \\
\tau_{3}=x_{3}^{\frac{1}{3}} .
\end{array}\right.
$$

Step 3. The case of $n \in \mathbb{N}$.: As in steps 1 and 2, (6) is geometrically expressed as a $n$-simplex-shaped region $D_{n}$ surrounded by $n+1$ hyperplanes $\tau_{1}=0, \tau_{i}=\tau_{i+1}(i=$ $1, \ldots, n-1)$, and $\tau_{n}=1$ in $n$-dimensional space, where the hypervolume of $D_{n}$ is

$$
H_{n}=\frac{1}{n !} .
$$

The probability density function of $\tau_{n}$ is $f_{n}\left(\tau_{n}\right)=n \tau_{n}^{n-1}$ by the same reasoning as in steps 1 and 2 . Thus, the general equation of the variable transformation for obtaining $\tau_{i}$ is expressed as follows:

$$
\begin{aligned}
\tau_{i} & =\prod_{k=i}^{n} x_{k}^{\frac{1}{k}} \\
& = \begin{cases}x_{i}^{\frac{1}{i}} \tau_{i+1}, & \text { if } i=1,2, \ldots, n-1 \\
x_{i}^{\frac{1}{i}}, & \text { if } i=n .\end{cases}
\end{aligned}
$$

This result is summarized in the following theorem.

Theorem 1: If a random number $\boldsymbol{x}=\left(x_{1}, x_{2}, \ldots, x_{n}\right)$ which is uniformly distributed on $[0,1]^{n}$ is transformed into a random number $\boldsymbol{\tau}=\left(\tau_{1}, \tau_{2}, \ldots, \tau_{n}\right)$ in accordance with (24), then $\tau$ is also distributed uniformly in the region of (6).

Proof: The probability density function $f_{\tau}$ of $\tau$ is expressed as the following equation by applying the variable transformation formula for probability density functions [7]:

$$
\boldsymbol{f}_{\boldsymbol{\tau}}(\boldsymbol{\tau})=|\boldsymbol{J}(\boldsymbol{x} \rightarrow \boldsymbol{\tau})| \boldsymbol{f}_{\boldsymbol{x}}(\boldsymbol{x}(\boldsymbol{\tau})) .
$$

Here, $f_{\boldsymbol{x}}$ is the probability density function of $\boldsymbol{x}$ and $\boldsymbol{J}(\boldsymbol{x} \rightarrow \boldsymbol{\tau})$ is the Jacobian matrix of the transformation of variable $\boldsymbol{x}$ into $\boldsymbol{\tau}$. Since $\boldsymbol{x}$ is expressed as

$$
x_{i}=\left\{\begin{array}{cl}
\frac{\tau_{i}^{i}}{\tau_{i+1}^{i}}, & \text { if } i=1,2, \ldots, n-1 \\
\tau_{i}^{i}, & \text { if } i=n
\end{array}\right.
$$

by using (24), the Jacobian determinant becomes

$$
\begin{aligned}
& |\boldsymbol{J}(\boldsymbol{x} \rightarrow \boldsymbol{\tau})|=\left|\begin{array}{cccc}
\frac{\partial x_{1}}{\partial \tau_{1}} & \frac{\partial x_{1}}{\partial \tau_{2}} & \cdots & \frac{\partial x_{1}}{\partial \tau_{n}} \\
\frac{\partial x_{2}}{\partial \tau_{1}} & \frac{\partial x_{2}}{\partial \tau_{2}} & \cdots & \frac{\partial x_{2}}{\partial \tau_{n}} \\
\vdots & \vdots & \ddots & \vdots \\
\frac{\partial x_{n}}{\partial \tau_{1}} & \frac{\partial x_{n}}{\partial \tau_{2}} & \cdots & \frac{\partial x_{n}}{\partial \tau_{n}}
\end{array}\right| \\
& =\left|\begin{array}{cccc}
\frac{1}{\tau_{2}} & -\frac{\tau_{1}}{\tau_{2}^{2}} & & 0 \\
\frac{2 \tau_{2}}{\tau_{3}^{2}} & -\frac{2 \tau_{2}^{2}}{\tau_{3}^{3}} & & 0 \\
\boldsymbol{O} & \ddots & \ddots \dot{\tau}_{n-1}^{n-2} & -\frac{(n-1) \tau_{n-1}^{n-1}}{\tau_{n}^{n-2}} \\
& & \frac{\left(n \tau_{n}^{n-1}\right.}{\tau_{n}^{n-1}}
\end{array}\right| \\
& =\frac{1}{\tau_{2}} \times \frac{2 \tau_{2}}{\tau_{3}^{2}} \times \cdots \times \frac{(n-1) \tau_{n-1}^{n-2}}{\tau_{n}^{n-1}} \times n \tau_{n}^{n-1}
\end{aligned}
$$

Additionally, from (24), it is apparent that (6) is correct. By substituting (27) into (25), $\boldsymbol{f}_{\boldsymbol{\tau}}(\boldsymbol{\tau})$ becomes

$$
\boldsymbol{f}_{\boldsymbol{\tau}}(\boldsymbol{\tau})= \begin{cases}n !, & \text { if } 0 \leq \tau_{1} \leq \cdots \leq \tau_{n} \leq 1 \\ 0, & \text { otherwise. }\end{cases}
$$

Thus, the theorem is true. Moreover, since the hypervolume of the region $0 \leq \tau_{1} \leq \cdots \leq \tau_{n} \leq 1$ is $H_{n}=1 / n$ !, the entire probability is 1 .

Finally, $\hat{\theta}_{\text {ref }}(t)$ is expressed as $\hat{\theta}_{\text {ref }}(t ; \boldsymbol{x}, \boldsymbol{w})$ by using $\boldsymbol{x}=$ $\left(x_{1}, \ldots, x_{n}\right)$ and $\boldsymbol{w}=\left(w_{1}, \ldots, w_{n}\right)$, which define $\hat{\theta}_{\text {ref }}(t)$.

\section{FORMULATION OF OPTIMIZATION PROBLEM}

An overview of the mesh setting for the CFD simulation is shown in Fig. 6, and the parameters of the mesh are listed in Table I. As seen in Fig. 6, the analytical region covers only the upper part of the bottle since the fluidity of the lower part is low. The fluid used in this problem is water at $20^{\circ} \mathrm{C}$, whose properties are listed in Table II. The volume of the fluid is set to an amount that lets the surface of the liquid reach $0.02[\mathrm{~m}]$ from the top of the bottle. The finish time of the simulation is $1.0[\mathrm{~s}]$, and the time interval for data acquisition is $0.001[\mathrm{~s}]$. Conveyance of the bottle is simulated by applying virtual angular velocities to the bottle, including gravitational acceleration of $-9.81\left[\mathrm{~m} / \mathrm{s}^{2}\right]$ in the direction of 


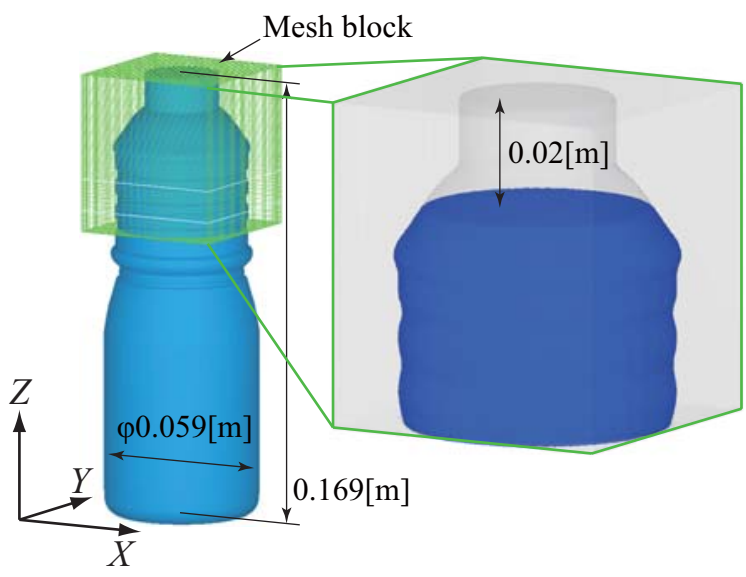

Fig. 6. Mesh settings for CFD simulation and rendered image

TABLE I

Mesh PARAMETERS

\begin{tabular}{|c|c|c|}
\hline Block & Cell size $[\mathrm{m}]$ & Number of cells \\
\hline $\mathrm{X}$ axis & 0.001 & 54 \\
\hline $\mathrm{Y}$ axis & 0.001 & 54 \\
\hline $\mathrm{Z}$ axis & $0.001-0.002$ & 52 \\
\hline \multicolumn{2}{|c|}{ Total number of cells } & 151632 \\
\hline
\end{tabular}

the $\mathrm{Y}$ axis. Based on the above settings, the computation time per simulation is about $30 \mathrm{~min}$.

Let the number of points that define a motion curve $\theta_{\text {ref }}(t)$ be $n=4$ in order to increase the flexibility of $\theta_{\text {ref }}(t)$. The rotation angle is $\theta_{\mathrm{f}} \approx 0.349[\mathrm{rad}]$, and the motion time is $t_{\mathrm{f}}=0.45[\mathrm{~s}]$. Thus, the actual angular displacement $\theta(t)$ of the wheel is determined from $\theta(t ; \boldsymbol{x}, \boldsymbol{w})$ by using (1), (8)(19), and (24). The first-, second- and third-order differentials of $\theta(t ; \boldsymbol{x}, \boldsymbol{w})$ are $\omega(t ; \boldsymbol{x}, \boldsymbol{w}), \alpha(t ; \boldsymbol{x}, \boldsymbol{w})$ and $j(t ; \boldsymbol{x}, \boldsymbol{w})$, respectively. We set the following constraint conditions on these functions:

$$
\left\{\begin{aligned}
& \min _{t} \omega(t ; \boldsymbol{x}, \boldsymbol{w}) \geq 0[\mathrm{rad} / \mathrm{s}] \\
& \max _{t}|\alpha(t ; \boldsymbol{x}, \boldsymbol{w})| \leq 12\left[\mathrm{rad} / \mathrm{s}^{2}\right] \\
& \max _{t}|j(t ; \boldsymbol{x}, \boldsymbol{w})| \leq 300\left[\mathrm{rad} / \mathrm{s}^{3}\right] .
\end{aligned}\right.
$$

In the optimization, the motion curve is evaluated by considering the maximum level $D_{\max }$ of the liquid $0.6[\mathrm{~s}]$ after starting the conveyance. $d_{\max }(t)$ is given by

$$
d_{\max }(t)=\max _{X, Y} d(X, Y, Z, t),
$$

and $D_{\max }$ is given by,

$$
D_{\max }=\max _{0.6 \leq t \leq 1} d_{\max }(t) .
$$

TABLE II

Properties OF WATER AT $20^{\circ} \mathrm{C}$

\begin{tabular}{|c|c|}
\hline Density & $1000\left[\mathrm{~kg} / \mathrm{m}^{3}\right]$ \\
\hline Viscosity & $0.001[\mathrm{~Pa} \cdot \mathrm{s}]$ \\
\hline Surface tension & $0.73[\mathrm{~N} / \mathrm{m}]$ \\
\hline Contact angle & $90[\mathrm{deg}]$ \\
\hline
\end{tabular}

TABLE III

PARAMETERS FOR REALGA

\begin{tabular}{|c|c|}
\hline Maximum number of individuals & 500 \\
\hline Population size & 20 \\
\hline Number of elite individuals & 19 \\
\hline Selection method & Roulette selection \\
\hline Crossover method & BLX- $\alpha(\alpha=0.2)$ \\
\hline
\end{tabular}

Here, $d(X, Y, Z, t)$ is the level of the liquid in each cell at position $(X, Y, Z)$ and time $t . d(X, Y, Z, t)$ is always constant regardless of $Z$ if $X, Y$, and $t$ are constant.

A RealGA is used for the optimization. The parameters for the RealGA are listed in Table III.

Finally, the optimization problem is defined by the following equation:

$$
\begin{array}{ll}
\text { Minimize } & D_{\max }(\boldsymbol{x}, \boldsymbol{w}) \\
\text { subject to } & \boldsymbol{x} \in[0,1]^{n} \\
& \boldsymbol{w} \in[0,1]^{n} \\
& \min _{t} \omega(t ; \boldsymbol{x}, \boldsymbol{w}) \geq 0 \\
& \max _{t}|\alpha(t ; \boldsymbol{x}, \boldsymbol{w})| \leq 12 \\
& \max _{t}|j(t ; \boldsymbol{x}, \boldsymbol{w})| \leq 300 .
\end{array}
$$

This is a problem that entails obtaining a motion curve that minimizes residual vibration at the surface of a liquid after its conveyance.

\section{RESULTS}

\section{A. Optimization Results}

The computation time for the optimization was about 250 $\mathrm{h}$ when using a PC with an Intel Core 2 Quad processor $(2.83 \mathrm{GHz})$.

The optimized solution is listed in Table IV, and the waveforms of the optimized motion curve and the modified sine curve are shown in Fig. 7 for comparison. Furthermore, the waveforms of each $d_{\max }(t)$ as calculated with (30) are shown in Fig. 8. The values of these curves as calculated from (31) are $0.0410[\mathrm{~m}]$ and $0.0452[\mathrm{~m}]$, respectively.

Fig. 7 shows that all of the maximum values of $\omega(t)$, $|\alpha(t)|$, and $|j(t)|$ of the optimized motion curve are slightly greater than those of the modified sine curve. Therefore, as shown in Fig. 8, the amplitude of vibration of the liquid in the case of the optimized motion curve during conveyance until $t=0.45[\mathrm{~s}]$ is larger than that of the modified sine curve. However, the optimized motion curve exhibits almost no residual vibration after conveyance $(t>0.45[\mathrm{~s}])$ and thus constitutes a positive and favorable result.

TABLE IV

OPTIMIZED SOLUTION

\begin{tabular}{|c|c|c|c|c|}
\hline$i$ & 1 & 2 & 3 & 4 \\
\hline$x_{i}$ & 0.6174 & 0.5629 & 0.1854 & 0.1632 \\
\hline$\left(\tau_{i}\right)$ & $(0.1679)$ & $(0.2719)$ & $(0.3624)$ & $(0.6356)$ \\
\hline$w_{i}$ & 0.3698 & 0.5798 & 0.8152 & 0.5269 \\
\hline
\end{tabular}




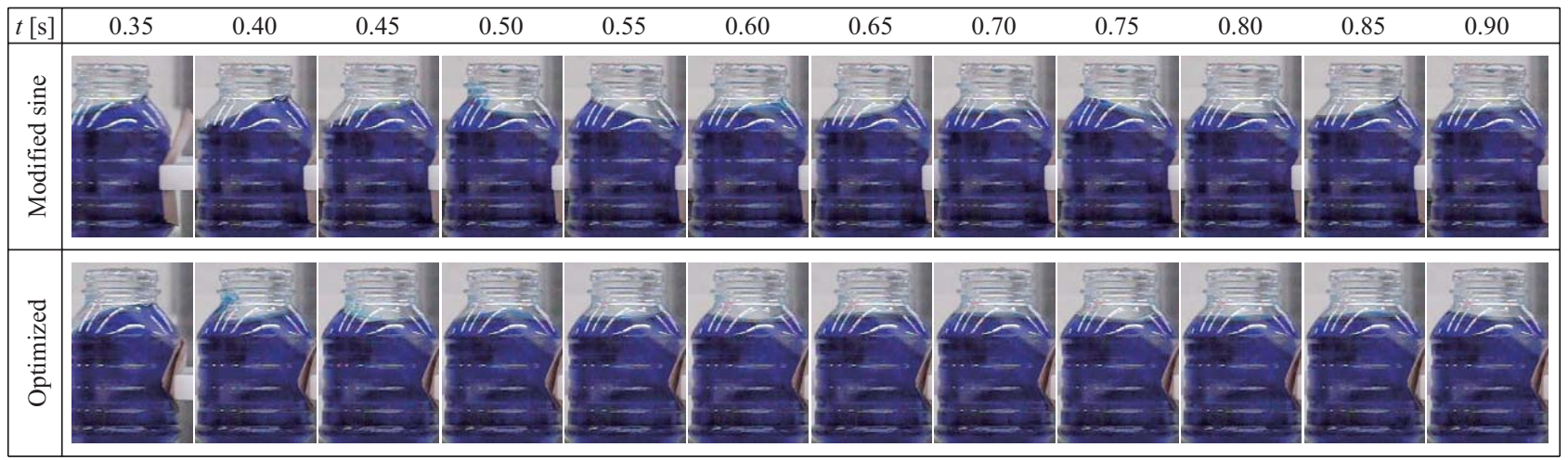

Fig. 9. Experimental results
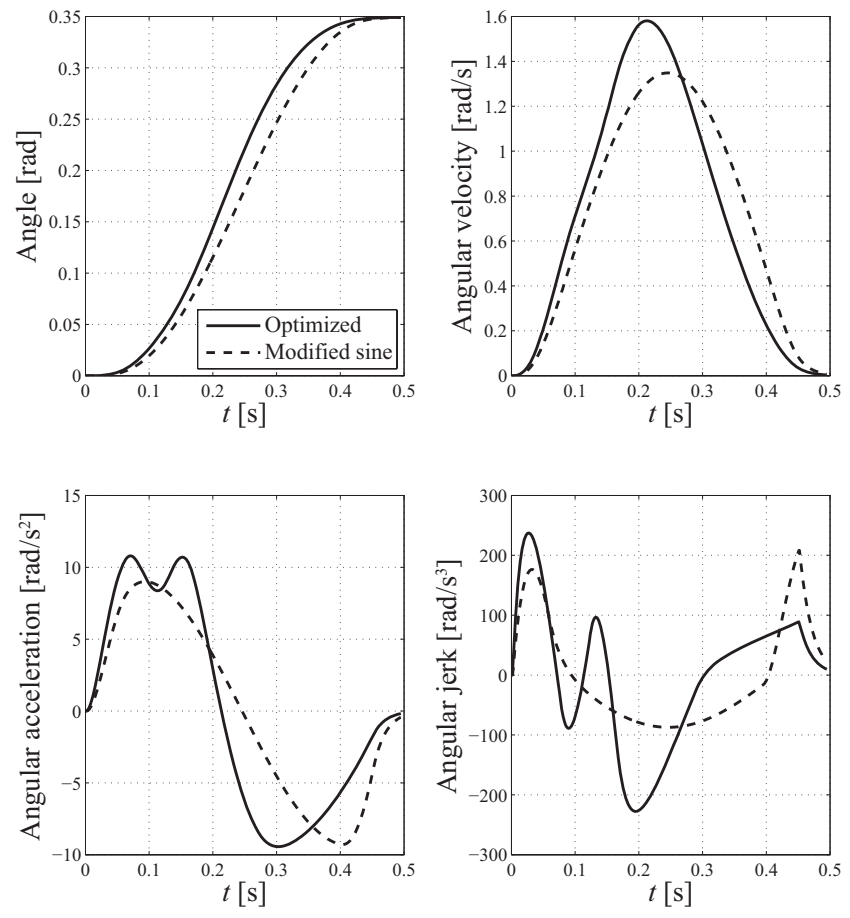

Fig. 7. Respective angle, angular velocity, angular acceleration, and angular jerk of the optimized motion curve and the modified sine curve

\section{B. Experimental Results}

We performed experiments by using an actual intermittent conveyance bottling machine. Photographs showing the behavior of liquid in bottles after they had been conveyed following the optimized motion curve and modified sine curve are shown in Fig. 9. The experimental results show that conveyance with the optimized motion curve can reduce residual vibration at the surface of a liquid, in accordance with the simulation results.

\section{CONCLUSIONS}

An optimized motion curve which minimizes residual vibration at the surface of a liquid in a bottle after it has been conveyed by an intermittent conveyance bottling machine has been obtained by using a CFD simulator and a RealGA. Both the simulation results and the experimentation results have shown that the optimized motion curve can reduce

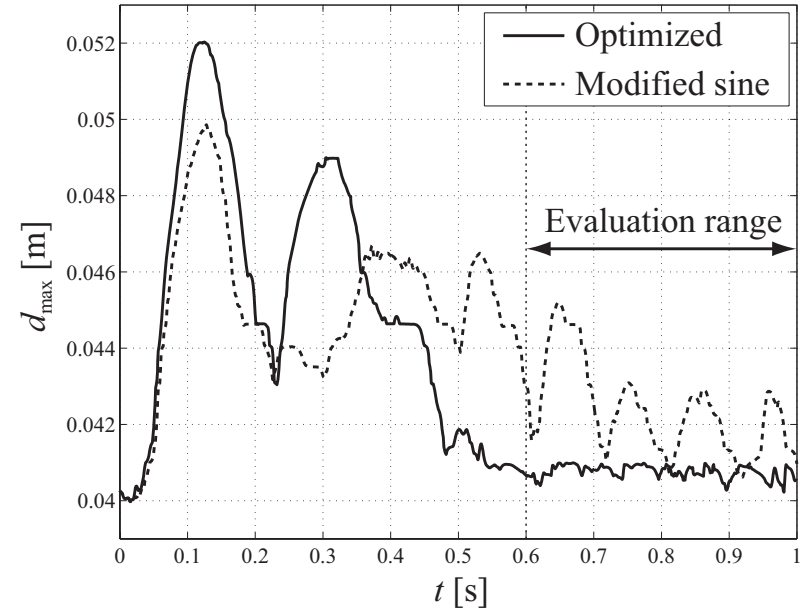

Fig. 8. Waveform of the maximum level of the liquid for the optimized motion curve and the modified sine curve

residual vibration at the surface of the liquid. Additionally, variable transformations that transform independent variables into variables with magnitude relations have been proposed and have been proved mathematically.

\section{REFERENCES}

[1] Y. Yoshida, M. Hamaguchi and T. Taniguchi, "Damping path design for liquid container transferred with wheeled mobile robot along multiple turn sections," in Proc. 17th World Congr. Int. Federation of Automatic Control, Seoul, Korea, 2008, pp. 12667-12672.

[2] Y. Kuriyama, K. Yano and M. Hamaguchi, "Trajectory planning for meal assist robot considering spilling avoidance," in 2008 IEEE Multiconf. Systems and Control (17th IEEE Conf. Control Applications), San Antonio, TX, 2008, pp. 1220-1225.

[3] Y. Kuriyama, S. Hayashi, K. Yano and M. Watanabe, "Solution search algorithm for a CFD optimization problem with multimodal solution space," in Proc. 48th IEEE Conf. Decision and Control, Shanghai, China, 2009, pp. 5556-5561.

[4] K. Kanazawa and K. Yano, "Computational fluid dynamics optimization of shape of sprue for die casting considering product quality," in Proc. 49th IEEE Conf. Decision and Control, Atlanta, GA, 2010, pp. 39083913.

[5] A. H. Wright, "Genetic algorithms for real parameter optimization," in Fundations of genetic algorithms, San Mateo, CA: Morgan Kaufmann Publishers, 1991, pp. 205-218.

[6] D. Kincaid and W. Cheney, "Approximating functions," in Numerical analysis: mathematics of scientific computing, 3rd ed. Providence, RI: AMS, 2002, pp. 349-354.

[7] Y. Fujikoshi, V. V. Ulyanov and R. Shimizu, "Multivariate normal and related distributions," in Multivariate statistics: high-dimensional and large-sample approximations, Danvers, MA: Wiley, 2010, pp. 1-6. 